\title{
Caso 1/2007 - Enterorragia e Choque em Mulher de 69 Anos Portadora de Estenose Mitral Reumática com Fibrilação Atrial Crônica
}

Case 1/2007 - Enterorrhagia and Shock in a 69 Year-old Woman with Rheumatic Mitral Stenosis and Chronic Atrial Fibrillation

Rafael Amorim Belo Nunes e Luiz Alberto Benvenuti

Instituto do Coração do Hospital das Clínicas - FMUSP - São Paulo, SP

Mulher de 69 anos de idade, portadora de estenose da valva mitral de etiologia reumática, procurou atendimento médico por dispepsia e enterorragia.

Os sintomas se iniciaram aos 37 anos de idade, quando apresentou dispnéia rapidamente progressiva até ocorrer em repouso. Nessa ocasião foi feito o diagnóstico de estenose da valva mitral, tendo sido medicada com digital e diurético, com melhora do quadro. Aos 40 anos de idade, foi submetida a comissurotomia mitral (abril/1972).

Evoluiu sem sintomas por 14 anos, fazendo seguimento no Hospital das Clínicas desde o quinto ano após a cirurgia. O exame físico do seu primeiro atendimento revelou freqüência cardíaca 56 bpm e pressão arterial 110x70 $\mathrm{mmHg}$ e hiperfonese de primeira bulha em área mitral. $\mathrm{O}$ eletrocardiograma era normal.
Após 14 anos, voltou a apresentar dispnéia intensa, que a levou a procurar atendimento médico de emergência (1986). Foi diagnosticada hipertensão arterial (pressão arterial 180x130 mmHg). O ecocardiograma (24/7/86) demonstrou fusão comissural sem estenose mitral (tab. 1). No final desse ano (outubro/1986), apresentou hemiparesia direita que durou horas. O eletrocardiograma revelou fibrilação atrial.

Foi feito o diagnóstico de acidente vascular cerebral e iniciado tratamento crônico com varfarina. Após o episódio inicial de dispnéia, fazia uso de hidroclorotiazida e amilorida (50 mg/5 mg), 0,25 mg digoxina e 2,5 mg de varfarina, e apresentava dispnéia aos esforços moderados.

Em 1993, queixou-se de dores articulares em mãos e pés, dores no tórax e dor lombar. A avaliação reumatológica na ocasião incluiu a pesquisa de anticorpos antinucleares e

\begin{tabular}{|c|c|c|c|c|c|c|}
\hline & Jul/86 & Jul/90 & Abr/97 & Nov/98 & Jul/00 & Abr/01 \\
\hline Septo & 7 & 8 & 8 & 8 & 9 & 9 \\
\hline Parede posterior & 7 & 8 & 8 & 8 & 9 & 9 \\
\hline DDVE & $\mathrm{n}$ & $\mathrm{n}$ & 46 & 42 & 41 & 41 \\
\hline DSVE & $\mathrm{n}$ & $\mathrm{n}$ & 31 & 25 & 26 & 31 \\
\hline FEVE & $\mathrm{n}$ & $\mathrm{n}$ & 0,69 & 0,78 & 0,73 & 0,71 \\
\hline Aorta & 29 & 29 & 29 & 29 & 29 & 31 \\
\hline $\mathrm{AE}$ & 44 & 44 & 49 & 54 & 56 & 58 \\
\hline VD & $\mathrm{n}$ & $\mathrm{n}$ & 20 & 24 & Aumentado & Aumentado \\
\hline Grad mitral & - & - & 3 & 4 & - & - \\
\hline Área Mitral & - & - & 1,7 & 1,7 & 1,9 & 1,5 \\
\hline V. tricúspide & I. leve & I. leve & I. Leve & I. Mod. & I. Mod. & I. Acent. \\
\hline
\end{tabular}

\section{Palavras-chave}

aterosclerose sistêmica, enterorragia, estenose mitral reumática, fibrilação atrial crônica.

Editor da Seção: Alfredo José Mansur (ajmansur@incor.usp.br)

Editores Associados: Desidério Favarato (dclfavarato@incor.usp.br)

Vera Demarchi Aiello (anpvera@incor.usp.br)

Correspondência: Vera Demarchi Aiello •

InCor - Av. Dr. Enéas de Carvalho Aguiar, 44 - 05403-000 - São Paulo, SP

E-mail: anpvera@incor.usp.br 


\section{Correlação Anatomoclínica}

do fator reumatóide, revelando-se negativa. Foram feitos os diagnósticos de dor de etiologia miofascial e artrite de etiologia a ser esclarecida.

Oito anos após o primeiro episódio, apresentou novamente hemiparesia direita transitória, que regrediu em horas (abril/1994). Foi ajustada a medicação.

Cerca de um ano após, em investigação para perda de peso (1995), foi feito o diagnóstico de diabete melito (1995) e inicialmente tratada com sulfoniluréia, e depois adicionada insulina NPH.

Aos 62 anos de idade, queixou-se de dor precordial e piora da dispnéia. $\mathrm{O}$ exame físico não mostrou informações adicionais. O eletrocardiograma (5/7/99) revelou fibrilação atrial, freqüência cardíaca de 130 bpm; baixa voltagem dos complexos QRS no plano frontal, SÂQRS $0^{\circ}$ para trás, sinais indiretos de sobrecarga atrial direita (sinal de PeñalozaTranchesi) e alterações difusas de repolarização ventricular (fig. 1).

Os exames laboratoriais (agosto/99) revelaram 13,7 g/dl de hemoglobina, hematócrito 41\%, 0,8 mg/dl de creatinina, glicemia $156 \mathrm{mg} / \mathrm{dl}$, colesterol total de $198 \mathrm{mg} / \mathrm{dl}$, HDLcolesterol de $52 \mathrm{mg} / \mathrm{dl}$, LDL-colesterol de $129 \mathrm{mg} / \mathrm{dl}$ e triglicérides de $85 \mathrm{mg} / \mathrm{dl}$.

Foi submetida a cateterismo cardíaco que não demonstrou gradiente entre a pressão de oclusão pulmonar e a pressão diastólica final de ventrículo esquerdo (tab. 2). A cineangiocoronariografia revelou artérias coronárias sem lesões obstrutivas, ventrículo esquerdo com movimentação normal na ventriculografia.

A medicação foi ajustada para $50 \mathrm{mg}$ de atenolol, 0,25 mg de digoxina, associação hidroclorotiazida/amilorida (50/5 mg), 5,125 mg de varfarina, e insulina NPH 27 unidades pela manhã e 27 unidades à noite.

Dois anos mais tarde, houve piora da dispnéia, que passou a ser desencadeada pelos mínimos esforços (abril/2001). O ecocardiograma (abril/2001; tab. 1) revelou estenose mitral moderada e insuficiência tricúspide intensa.

Foi indicado novo estudo cineangiográfico, com vistas ao tratamento cirúrgico das lesões valvares. O estudo hemodinâmico e cineangiográfico (19/1/01) revelou hipertensão pulmonar e ausência de lesões obstrutivas em coronárias (tab. 2).

Enquanto aguardava internação para cirurgia, houve aparecimento de úlcera infectada em membro inferior direito, o que fez postergar mais ainda a internação para a cirurgia para tratamento das valvopatias. Nesse período (julho/2001), estava no toalete quando sentiu fraqueza nos membros inferiores e sofreu queda e fratura de membro superior direito.

Aos 65 anos de idade, procurou atendimento médico de emergência por dor epigástrica, inapetência e náuseas $(11 / 2 / 2002)$

\begin{tabular}{lcc|}
\hline Data & $\mathbf{5 / 8} / \mathbf{9 9}$ & $\mathbf{1 9 / 6 / 0 1}$ \\
\hline Átrio direito (média) & 6 & 8 \\
\hline VD (Sist/Diastini/Diastfinal) & $36 / 0 / 15$ & $51 / 0 / 8$ \\
Art. Pul. (Sist/Diast/média) & $36 / 12 / 20$ & $51 / 16 / 29$ \\
\hline Oclusão pul. (média) & 12 & 18 \\
\hline VE (Sist/Diastini/Diastfinal) & $180 / 0 / 12$ & $170 / 0 / 15$ \\
\hline Aorta (Sist/Diast/média) & $180 / 80 / 110$ & $170 / 70 / 103$ \\
VD - ventrículo direito; Sist - sistólico; Diastini - diastólica inicial; Diast final \\
- diastólica final; Art. Pul. - artéria pulmonar; VE - ventrículo esquerdo. \\
\hline
\end{tabular}

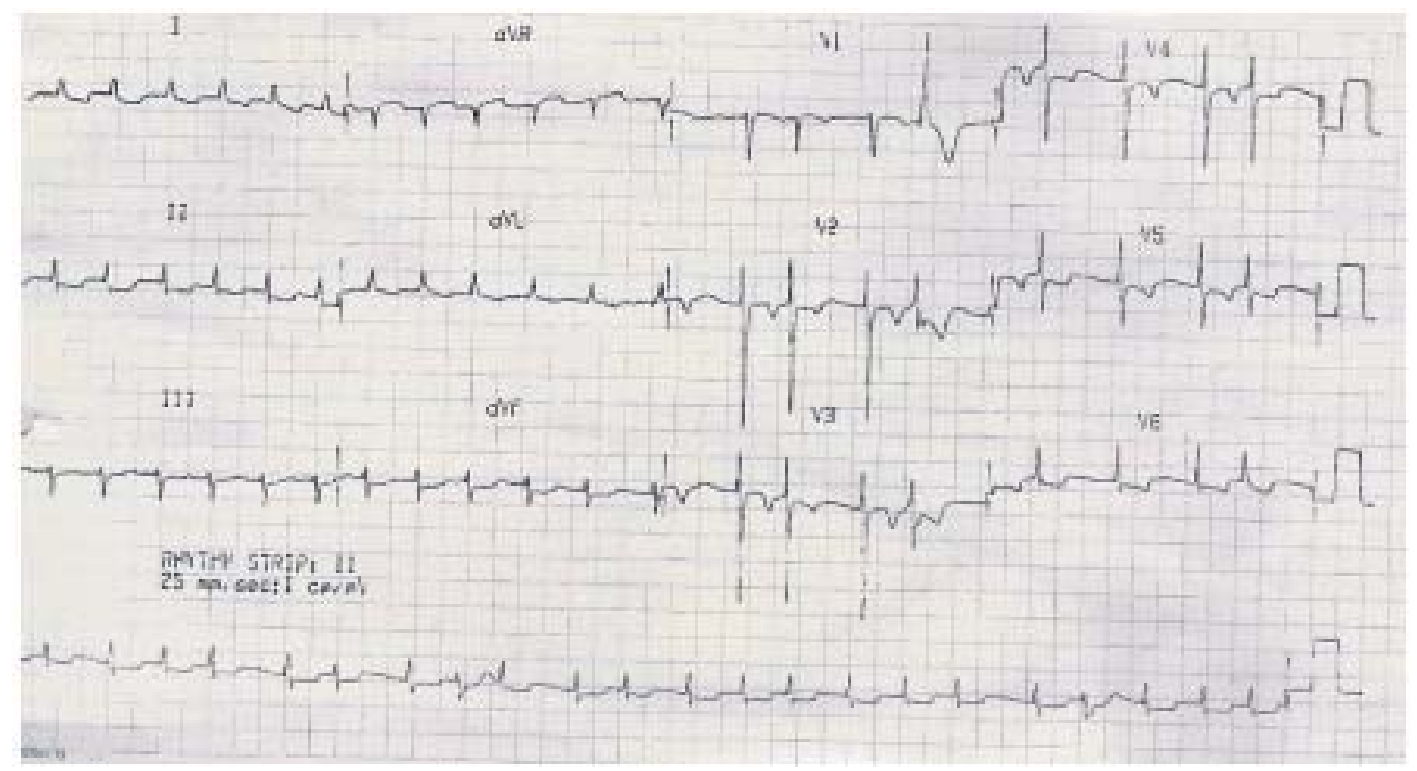

Fig. 1 - ECG - Fibrilação atrial, baixa voltagem dos complexos QRS no plano frontal, sinais indiretos de sobrecarga atrial direita (sinal de Peñaloza-Tranchesi) e alterações difusas de repolarização ventricular. 
O exame físico revelou freqüência cardíaca 110 bpm, pressão arterial de $120 \times 80 \mathrm{mmHg}$. A semiologia pulmonar não demonstrou ruídos adventícios. A semiologia cardíaca revelou bulhas arrítmicas e sopro sistólico em área tricúspide. O exame do abdome revelou abdome flácido, indolor, com ruídos hidroaéreos presentes. Os membros não apresentavam edema.

Os exames laboratoriais (11/2/2002) revelaram uréia de $204 \mathrm{mg} / \mathrm{dl}$, creatinina de 2,3 mg/dl, glicemia de $331 \mathrm{mg} / \mathrm{dl}$, sódio de $127 \mathrm{mEq} / \mathrm{l}$, potássio de $5,7 \mathrm{mEq} / \mathrm{l}$, hemoglobina de $18 \mathrm{~g} / \mathrm{dl}$, hematócrito de 56\%, 21.600 leucócitos por $\mathrm{mm}^{3} \mathrm{e}$ 408.000 plaquetas por $\mathrm{mm}^{3}$.

Foi feito o diagnóstico de descompensação diabética de etiologia a ser esclarecida, acompanhada de insuficiência renal aguda. Foram iniciadas hidratação e insulina intravenosas com controle da hiperglicemia e da hiperpotassemia.

No dia seguinte, queixou-se de dor abdominal e fezes sanguinolentas. $\mathrm{O}$ abdome tornou-se difusamente doloroso à palpação, mais sensível em epigástrio, não havendo dor à descompressão brusca.

Os exames laboratoriais $(12 / 2 / 2002)$ revelaram: hemoglobina de $16,9 \mathrm{~g} / \mathrm{dl}$, hematócrito de $52 \%$, leucócitos $28.500 / \mathrm{mm}^{3}$ (8\% bastonetes, $85 \%$ segmentados, 3\% linfócitos e $4 \%$ monócitos), 356.000/ $\mathrm{mm}^{3}$ plaquetas; glicemia $220 \mathrm{mg} /$ dl, creatinina 1,9 mg/dl, uréia $201 \mathrm{mg} / \mathrm{dl}$, sódio $144 \mathrm{mEq} / \mathrm{l}$ e potássio 2,9 mEq/l e tempo de protrombina (INR) de 2,17.

A endoscopia digestiva alta (12/2/2002) revelou estase gástrica intensa, atrofia moderada de mucosa gástrica, gastrite erosiva leve e úlceras antrais ativas com sinais tardios de sangramento prévio.

A paciente evolui com sinais de desidratação e hipotensão. A freqüência cardíaca era 100 bpm e a pressão arterial, de $80 \times 40 \mathrm{mmHg}$. Foram administrados ceftriaxona, reposição volêmica e dopamina intravenosos.

O coagulograma revelou INR de 9,8, a creatinina se elevou para $3 \mathrm{mg} / \mathrm{dl}$, a uréia era de $199 \mathrm{mg} / \mathrm{dl}$, a glicemia, de $124 \mathrm{mg} /$ dl, o leucograma revelou 13.800 leucócitos $/ \mathrm{mm}^{3}$ (mielócitos $1 \%$,metamielócitos $11 \%$, bastões $42 \%$, segmentados $28 \%$, linfócitos 8\% e monócitos 10\%).

A paciente continuou em choque e faleceu (13/2/2002).

\section{Aspectos clínicos}

Trata-se de mulher de 69 anos de idade, portadora de estenose da valva mitral, que procurou atendimento médico de emergência com dor epigástrica aguda e náuseas, evoluindo nos dias subseqüentes com dor abdominal difusa, sangramento digestivo baixo e instabilidade hemodinâmica. A paciente apresentava as seguintes comorbidades: fibrilação atrial, hipertensão arterial e antecedente de dois acidentes cerebrovasculares.

Ao dar entrada no pronto-socorro, ela se encontrava desidratada e taquicárdica. Os exames físico abdominal, pulmonar e cardiovascular eram inespecíficos. No entanto, no dia seguinte a paciente evoluiu com distensão abdominal e evacuação com fezes sanguinolentas. Os exames laboratoriais demonstravam altos níveis glicêmicos, uréia e creatinina elevadas com relação creatinina/uréia superior a 1/40, sugerindo insuficiência renal aguda de origem pré-renal, hipercalemia e leucocitose acentuada. Com a hipótese diagnóstica de diabete melito descompensado iniciou-se tratamento específico no serviço de emergência.

O diagnóstico diferencial da dor abdominal aguda associada a repercussões sistêmicas é amplo e inclui afecções primárias abdominais, abrangendo processos inflamatórios, obstruções intestinais, perfurações de vísceras ocas e distúrbios vasculares, assim como afecções extra-abdominais que podem cursar com acometimento secundário do aparelho digestivo, como alterações metabólicas (exemplo: cetoacidose diabética) e intoxicações exógenas, entre outros.

No caso da nossa paciente, inicialmente não havia sinais localizatórios abdominais como peritonismo, defesa abdominal involuntária, e os ruídos hidroaéreos estavam presentes, suscitando uma possível causa metabólica como razão da sintomatologia abdominal. No entanto, apesar da realização de reposição volêmica e controle dos níveis glicêmicos com insulinoterapia, a paciente evolui rapidamente com choque, necessitando de agente vasoativo e intubação orotraqueal. Na hipótese de choque séptico associado à descompensação diabética, introduziu-se ao esquema terapêutico antibiótico de amplo espectro. Entretanto, um possível foco não foi determinado, impossibilitando a exclusão de doenças que necessitassem de intervenção cirúrgica.

Na vigência da tríade clássica: dor abdominal desproporcional aos achados clínicos abdominais, fezes sanguinolentas e fonte embólica óbvia, como fibrilação atrial ou infarto do miocárdio recente, o diagnóstico de isquemia mesentérica aguda deve ser considerado

A isquemia mesentérica aguda resulta da oclusão da artéria mesentérica superior e, menos freqüentemente, da artéria mesentérica inferior, habitualmente como complicação de um evento embólico ou doença aterosclerótica mesentérica. $\mathrm{Na}$ embolia mesentérica, o êmbolo origina-se geralmente no átrio esquerdo ou ventrículo esquerdo, e ocasionalmente na aorta torácica ou abdominal. Os sintomas mais comuns, segundo estudo de Park e cols. ${ }^{2}$, são dor abdominal (95\% dos pacientes), seguida de náuseas em 44\% dos pacientes. Diarréia com sangue ou hematoquezia estão presentes em $16 \%$ dos pacientes, mas enterorragia maciça é incomum. A leucocitose é achado laboratorial freqüente, estando presente em mais de $90 \%$ dos pacientes. A isquemia mesentérica está associada a altos índices de mortalidade (70\% a 90\%), principalmente quando associada ao diagnóstico tardio, com a ocorrência de necrose intestinal e complicações sistêmicas como septicemia e síndrome inflamatória sistêmica ${ }^{3}$. Como os sintomas são inespecíficos, geralmente o diagnóstico depende de alta suspeição clínica e muitas vezes não é realizado antes do óbito.

A paciente em questão apresentava vários fatores de risco para embolização sistêmica, o que contribui para hipótese diagnóstica de isquemia mesentérica aguda.

Antes do advento da terapia com anticoagulantes, 25\% dos óbitos em pacientes com estenose mitral estavam relacionados a embolizações sistêmicas ${ }^{4,5}$. Ainda mais, a associação entre estenose mitral e fibrilação atrial confere 


\section{Correlação Anatomoclínica}

um risco de embolização sistêmica entre $4 \%$ e $6 \%$ ao ano. O uso de anticoagulantes reduz, mas não exclui, o risco de complicações cardioembólicas. Segundo trabalho de Chiang e cols. ${ }^{6}$, os preditores mais importantes de embolização sistêmica são idade, tamanho do átrio esquerdo e presença de fibrilação atrial. A paciente era idosa, portadora de fibrilação atrial permanente e vinha evoluindo com aumento do átrio esquerdo $(5,8 \mathrm{~cm})$.

Apesar de menos de $10 \%$ das hemorragias baixas resultarem de sangramento originado acima do ângulo de Treitz, a endoscopia digestiva alta na paciente em questão demonstrou a presença de sangramento difuso da mucosa gástrica ${ }^{7}$. Sangramentos gástricos podem ser causa de instabilidade hemodinâmica e choque, especialmente quando de origem arterial (exemplo: lesão arterial associada a úlcera péptica). Erosões da mucosa gástrica e hemorragia subepitelial (gastrite de estresse) normalmente são encontradas em pacientes críticos, e menos de $2 \%$ desses sangramentos apresentam repercussão clínica relevante. As altas taxas de mortalidade nesse grupo estão mais relacionadas à doença de base do que ao sangramento digestivo. Hemorragias digestivas em pacientes críticos também podem ocorrer na vigência de alterações da coagulação (exemplo: coagulopatia da septicemia, coagulação intravascular disseminada, uso de fármacos anticoagulantes). Portanto, consideramos que o sangramento digestivo seja possivelmente uma complicação secundária à doença aguda e ao estado crítico vigente.

(Dr. Rafael Amorim Belo Nunes)

\section{Hipótese diagnóstica}

Isquemia mesentérica aguda de origem cardioembólica, em paciente portadora de estenose valva mitral de etiologia reumática e fibrilação atrial crônica.

(Dr. Rafael Amorim Belo Nunes)

\section{Necropsia}

$\mathrm{Na}$ abertura do abdome, notou-se discreta ascite serohemorrágica. Havia infarto hemorrágico recente acometendo extensas áreas do tubo digestivo, especialmente o estômago e o intestino delgado, com contaminação bacteriana secundária (fig. 2). A aorta exibia aterosclerose moderada, com calcificação de placas, especialmente no segmento abdominal. Os rins apresentavam lesões isquêmicas crônicas, com infartos cicatrizados e em cicatrização, com atrofia parenquimatosa principalmente à direita. Havia áreas de infarto antigo, cicatrizado, em córtex cerebelar e nos lobos temporal e occiptal direito do cérebro.

O coração pesou $340 \mathrm{~g}$, havendo discreta hipertrofia de ambos os ventrículos. O átrio esquerdo apresentava pequeno aumento de volume, com espessamento endocárdico, notando-se pontos cirúrgicos antigos em seu teto. Não havia trombos cavitários. A valva mitral mostrava cúspides com acentuado espessamento fibroso e áreas focais de calcificação. Mostrava ainda evidências de procedimento cirúrgico prévio (comissurotomia). As cordas tendíneas estavam encurtadas e apresentavam áreas de fusão (fig. 3). As demais valvas cardíacas não apresentavam anormalidades e as artérias coronárias epicárdicas não exibiam lesões ateroscleróticas significativas. Os pulmões apresentavam evidências de congestão passiva crônica, com espessamento da parede das veias parenquimatosas.

(Dr. Luiz Alberto Benvenuti)

\section{Diagnósticos anatomopatológicos}

Aterosclerose sistêmica; infartos antigos, cicatrizados, dos rins e encéfalo; infarto hemorrágico recente do estômago e intestinos - necrose mesentérica (causa do óbito); estenose mitral reumática crônica (operada).

(Dr. Luiz Alberto Benvenuti)

\section{Comentários}

Caso de paciente do sexo feminino, com 69 anos de idade, portadora de estenose da valva mitral de etiologia reumática associada a fibrilação atrial crônica. Foi submetida previamente a cirurgia de comissurotomia da valva mitral, com sucesso.

Também era portadora de aterosclerose sistêmica, com infartos antigos dos rins e encéfalo, tendo apresentado necrose mesentérica com evolução fatal, caracterizada por extenso infarto hemorrágico do estômago e intestinos.

A lesão isquêmica do trato gastrointestinal pode ter conseqüências catastróficas, como no presente caso, sendo usualmente secundária a oclusão trombótica ou embólica arterial ou a baixo fluxo sangüíneo de leito arterial severamente acometido por aterosclerose ${ }^{8}$. No caso apresentado, apesar da existência de fibrilação atrial crônica associada à estenose mitral reumática, não encontramos trombos no interior do átrio esquerdo que justificassem eventual embolia de ramos arteriais mesentéricos. Portanto, apesar de não podermos afastar totalmente a possibilidade de embolia, cremos que o infarto intestinal seja conseqüente à doença aterosclerótica da aorta, da qual a paciente também era portadora.

A valvopatia reumática crônica pode estar associada à doença aterosclerótica, como ocorreu no presente caso. Apesar de não termos encontrado relatos de associação entre doença reumática e complicações da doença aterosclerótica da aorta e ramos mesentéricos, há descrições e estudos da concomitância entre valvopatia reumática crônica e doença aterosclerótica das artérias coronárias ${ }^{9,10}$.

(Dr. Luiz Alberto Benvenuti) 


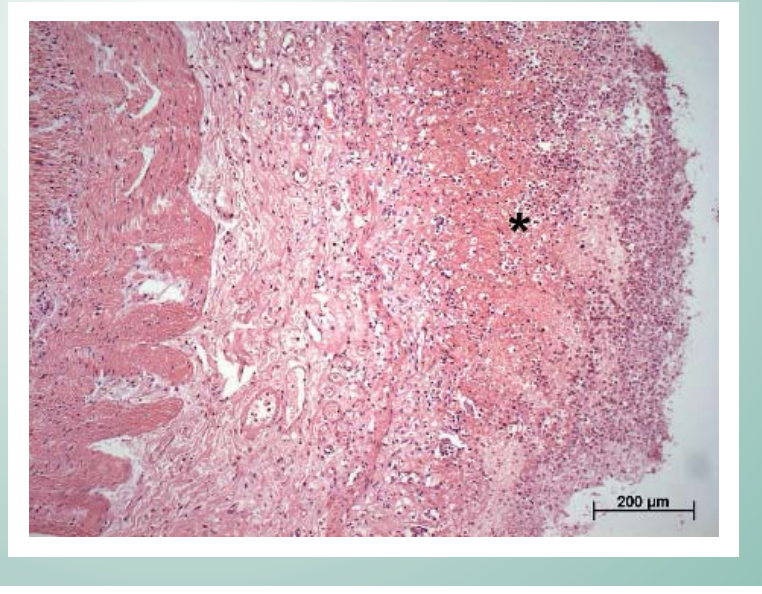

Fig. 2 - Corte histológico do intestino delgado evidenciando necrose hemorrágica da mucosa (asterisco). Hematoxilina-eosina.

\section{Referências}

1. Oldenburg WA, Lau LL, Rodenberg, TJ, Edmonds HJ, Burger CD. Acute Mesenteric Ischemia: A clinical review. Arch Intern Med 2003; 164: 1054-62

2. Park WM, Gloviczki P, Kenneth CJ, Hallet JW, Bower TC, Panneton JM, et al. Contemporary management of acute mesenteric ischemia: Factors asociated with survival. J Vasc Surg 2002; 35: 445-52.

3. Char DJ, Cuadra AS, Hines GL, Purtill W. Surgical Intervention for Acute Intestinal Ischemia: Experience in a Community Teaching Hospital. Vasc Endovasc Surg 2003; 37: 245-52.

4. Braunwald E, Zipes DP, Libby P. Heart Disease: A Textbook of Cardiovascular Medicine. 6.ed. Phyladelphia: W. B. Saunders; 2001: 1646.

5. Nielso GH, Galea EG, Houssack KF. Thromboembolic complications of mitral valve disease. Aust NZ J Med 1978; 8: 372

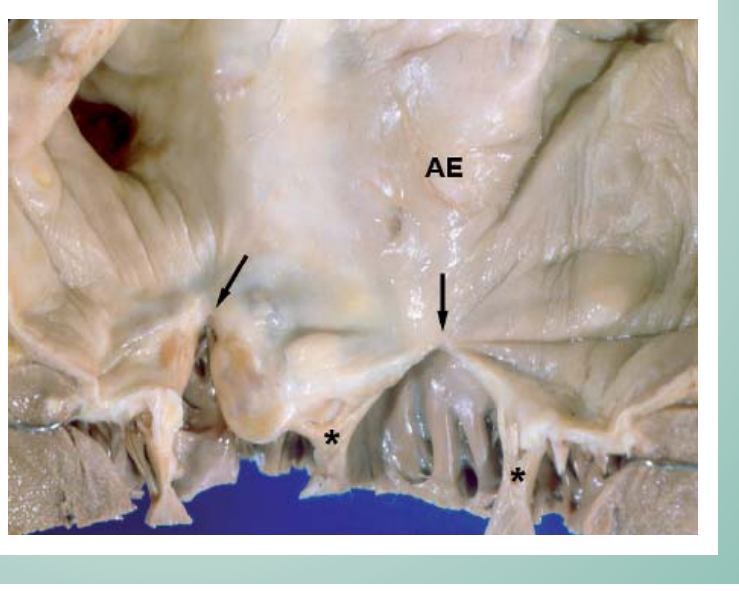

Fig. 3 - Visão da valva mitral e átrio esquerdo. Nota-se espessamento fibroso das cúspides e cordas tendíneas, com fusão e encurtamento das últimas (asteriscos). Há ainda sinais de valvotomia cirúrgica (setas) e espessamento do endocárdio do átrio esquerdo $(A E)$

6. Chiang CW, Lo SK, Ko YS, Cheng NJ, Lin PJ, Chang CH. Predictors of systemic embolism in patients with mitral stenosis. Ann Intern Med 1998; 128 : 885-9.

7. Wolfe MM, Sachs G. Acid suppression: optimizing therapy for gastroduodenal ulcer healing, gastroesofhageal reflux disease, and stress-related erosive syndrome. Gastroenterology 2000; 118(2 Suppl 1): S9-31.

8. Burns BJ, Brandt LJ. Intestinal ischemia. Gastroenterol Clin North Am 2003; 32: 1127-43.

9. Splugas E, Amer R, Barthe JE, Jara F. Distribution of significant coronary lesions in patients with rheumatic valvular heart disease. Study of 300 consecutive cases. Med Clin (Barc) 1980; 75: 112-4.

10. Salas-Lara VM, Rangel-Abundis A, Solorio-Meza S, Albarran-Lopez H. Assessment of a predictive index for coronary artery disease in patients with rheumatic valvular disease Cir Cir 2005:73:85-9. 